\title{
Stability Configurations of Electrons on a Sphere
}

An unsolved mathematical problem created by J. J. Thomson's efforts to visualize the nucleus of an atom is the determination of stable configurations of a given number $(n)$ of electrons bound to the unit sphere and interacting under mutual (newtonian) repulsion. Clearly, one such position would be that of lowest potential energy, but uniqueness and presence of symmetries are still unsettled questions. As an analytic procedure a stable configuration can most often be determined by a lucky guess supported by the explicitly calculated potential energy, checked to be a semi-definite quadratic form in the infinitesimal displacements from the conjectured configuration. On the other hand from the point of view of the computing machine a feasible method is to start with an "arbitrary" configuration and attain the minimum potential energy by descent. The former method (of infinitesimal displacements) was used by L. Föppl [1] to find stable configurations for all $n \leq 8$ and several larger $n$, some of which are summarized below in terms of "rings."

$$
\begin{aligned}
& 5=1+3+1 \\
& 8=1+3+3+1 \\
& 6=1+4+1 \\
& 10=1+4+4+1 \\
& 7=1+5+1 \\
& 12=1+5+5+1 \\
& 14=1+6+6+1 \text {. }
\end{aligned}
$$

The notation " $n=1+m+1$ " denotes one electron at each pole $(0,0, \pm 1)$ and $m$ electrons forming a regular polygon (or ring) on the equator $(z=0)$. The notation " $n=1+m+m+1$ " denotes one electron at each pole and two regular polygons of $m$ electrons at equal and opposite latitudes situated so that each electron in the upper hemisphere $(z>0)$ is antipodal to an electron in the lower hemisphere $(z<0)$. Stable configurations for $n=4,6,8,12$, 20 were seen by Föppl to correspond to the five regular solids. The first two omissions in Föppl's work were $n=9$ and $n=11$. The purpose of this study is to find stable configurations for these values of $n$ by descent, using the IBM 701. The principal new results (December 1955) are that $n=9$ leads to three rings of equilateral triangles with rotational symmetry, while $n=11$ is quite irregular, having planar but not rotational symmetry, on the basis of the numerical evidence submitted here.

We start with an "arbitrary" configuration standardized as $P_{1}{ }^{(0)}=(0,0,1)$, $P_{2}{ }^{(0)}=(0, .6,-.8), P_{3}(0)=(.6,0, .8), P_{t+4}^{0}=(\sin 2 \pi t /(n-3), \cos 2 \pi t /(n-3), 0)$, where $0 \leq t \leq n-4$, so as to avoid superimposing any artificial symmetry. More generally let us consider the main loop as consisting of one step in the descent, where the general configuration is given by $3 n$ quantities:

$$
P_{i}=\left(x_{i}, y_{i}, z_{i}\right), \quad 1 \leq i \leq n, \quad\left(x_{i}{ }^{2}+y_{i}{ }^{2}+z_{i}{ }^{2}=1\right) .
$$


The (tangential) force on the particle at $P_{i}$ is $\left(X_{i}, Y_{i}, Z_{i}\right)$ where

(2) $X_{i}=\sum_{i \neq j} \frac{\left(x_{i}-x_{j}\right)-x_{i}\left[\left(x_{i}-x_{j}\right) x_{i}+\left(y_{i}-y_{j}\right) y_{i}+\left(z_{i}-z_{j}\right) z_{i}\right]}{\left[\left(x_{i}-x_{j}\right)^{2}+\left(y_{i}-y_{j}\right)^{2}+\left(z_{i}-z_{j}\right)^{2}\right]^{3}}$, etc.,

and the point $P_{i}$ is moved to $\left(x_{i}{ }^{\prime}, y_{i}{ }^{\prime}, z_{i}{ }^{\prime}\right)$ where

$$
x_{i}^{\prime}=x_{i}+h X_{i}, \text { etc. }
$$

Here $h$ is a positive constant to be determined automatically as described later on. Then, replacing $P_{i}$ by $P_{i}(h)=\left(x_{i}(h), y_{i}(h), z_{i}(h)\right)$, where

$$
x_{i}(h)=x_{i}{ }^{\prime} /\left(x_{i}{ }^{2}+y_{i}{ }^{2}+z_{i}{ }^{\prime 2}\right), \text { etc., }
$$

we close the loop. The exit occurs when

$$
\xi=\max \left\{\left|x_{i}-x_{i}(h)\right|,\left|y_{i}-y_{i}(h)\right|,\left|z_{i}-z_{i}(h)\right|\right\}<\epsilon .
$$

The choice of "step-size" $h$ has to be made internally. We consider only those $h$ of the type $h=h_{0} \rho^{k}$ where $h_{0}$ is a positive parameter (initially .1 and variable with each circuit of the main loop), $\rho(>1)$ is a fixed ratio taken as $2^{\frac{1}{2}}$, and $k$ is an integer to be chosen. We call $V\left(=\sum_{i>j}\left|P_{i} P_{j}\right|^{-1}\right)$ the potential of the configuration $P_{i}$ and $V(h)$ that of the configuration $P_{i}(h)$. There are two possibilities. The first possibility is that $V\left(h_{0}\right)<V$. In that case we choose $h=h_{0}(k=0)$ unless $V\left(h_{0} \rho\right)<V\left(h_{0}\right)$, in which case we choose $k$ to be the minimum positive integer for which $V\left(h_{0} \rho^{t}\right)<V\left(h_{0} \rho^{t-1}\right), t=1,2, \cdots, k$, while $V\left(h_{0} \rho^{k+1}\right) \geq V\left(h_{0} \rho^{k}\right)$. (We must exclude (by programming) the possibility that $h$ be $\infty$ or that the $3 n$ corrected coordinates $x_{i}{ }^{\prime}, y_{i}{ }^{\prime}, z_{i}{ }^{\prime}$ be parallel to the force vectors $X_{i}, Y_{i}, Z_{i}$. This might happen accidentally when radius vectors parallel to the force vectors represent a configuration of lower potential energy than the configuration $P_{i}$.) Then $h=h_{0} \rho^{k}$ and $h$ replaces $h_{0}$. The second possibility is that $V\left(h_{0}\right) \geq V$. In this case, letting $k$ be the minimum positive integer for which $V\left(h_{0} \rho^{-k}\right)<V$, then $h=h_{0} \rho^{-k}$ with $h$ now replacing $h_{0}$. (Here again $h$ might become zero through round-off loss in the computation of $V(h)$, and this possibility must be excluded by programming.)

In practice, $h=h_{0}$ in at least $\frac{5}{6}$ of the cases; and when $h$ changed, it changed only by the factor $\rho^{ \pm 1}$. Generally $.2<h<.4$.

The program was coded in Speed Code III and read in from IBM instruction cards and floating decimal cards for $n, h, \rho, \epsilon$, etc. The use of a binary instruction deck cut the read-in time of 150 cards per minute to less than two minutes. The machine generated the $P_{i}{ }^{(0)}$ internally and, in cases of reruns, accepted the last $P_{i}$ and $h$ and modified entrance instruction from correction cards. Each major (descent) loop took about $.3 n^{2}$ seconds (slightly longer if $h$ was changed by the loop). At the end of each loop the machine loaded the pairs $h, V(h)$ in an output block of the memory, dumping the pairs 25 at a time to provide some means of observation. The machine also loaded the $3 n+3$ values $\xi, V, h, P_{i}$ on tape, although there was no occasion to dump the tape later on. As a further monitoring device the sensing switch $P$ was made available to print these $3 n+3$ values at 
the end of each loop when desired. At the exit, the residual $(h, V(h))$ pairs, $3 n+3$ aforementioned values, and the $n^{2}$ mutual distances $\left|P_{i} P_{j}\right|$ were printed out; and the $Q$ switch was used to bring the computation to the exit at the end of the current loop in case of shortage of time. Print-out time was used sparingly since each printing operation takes $5 / 3+2 l / 5$ seconds for $l$ lines of 5 words per line.

The descent described becomes badly oscillatory; and although we need not regard this fact as wholly unfavorable, we should see the descent in more conventional terms. (The oscillations simulate vibrations in that normal modes can be identified. This will be the subject of a more complete study later on.) We imagine the particle positions parametrized in terms of $2 n$ independent variables $q_{i}$ such as two of the three displacement coordinates of each point from equilibrium. We assume small displacements. Then some real, symmetric, and (presumably) positive, semi-definite matrix $\left\|a_{i j}\right\|$ exists such that $2 V=\sum a_{i j} q_{i} q_{j}$. The matrix has the eigenvalues $\lambda_{i}(\geq 0)$ and normal coordinates $Q_{i}$ for which $2 V=\sum \lambda_{i} Q_{i}{ }^{2}$. Thus equation (3) becomes approximately

$$
\Delta q_{i}=-h \partial V / \partial q_{i}=-h \sum a_{i j} q_{j}
$$

or

$$
\Delta Q_{i}=-h \lambda_{i} Q_{i}
$$

suggesting exponential decay rather than "dynamic" oscillation. The oscillatory behavior here is (presumably) caused by the large range of non-vanishing $\lambda_{i}$, or by the fact that no one $h$ can make all the "decay ratios" $\left|\left(Q_{i}+\Delta Q_{i}\right) / Q_{i}\right|$ $=\left|1-h \lambda_{i}\right|$ appreciably less than unity (for $\lambda_{i}>0$ ).

Certainly, for purposes of establishing a (conjectured) local minimum for $V$, it would be reasonable to check the $\lambda_{i}$. We are concerned more with the preliminary process of obtaining the initial conjecture in the face of very slow convergence. The procedure will be to cut the number of degrees of freedom, and (effectively) the range of $\lambda_{i}$ through conjectured symmetries. For instance when $n=9(n=11)$ for the initial configuration $P_{i}{ }^{(0)}$ described earlier $V=27.07665314$ $(=43.03629491)$ and after $32(51)$ circuits of the main loop $V$ had descended to the value 25.76006543 (40.59907213), stable to four (two) decimal places, while the oscillations in particle position, $\xi$, had the order of magnitude of $10^{-2}\left(10^{-1}\right)$ with no perceptible improvement. Yet a drawing on graph paper of the "final," i.e., the thirty-second (fifty-first) approximation for $n=9(n=11)$ displayed enough symmetry to enable us to cut down the number of degrees of freedom from 18 to 1 ( 22 to 5 ).

In particular, when $n=9$ it becomes graphically clear that the arrangement is $9=3+3+3$, i.e., an equilateral triangle on the equator with two symmetric equilateral triangles, one in each hemisphere, giving the nine points $(1,0,0)$, $\left(-\frac{1}{2}, \pm 3^{\frac{1}{3}} / 2,0\right),\left(\left(1-\alpha^{2}\right)^{\frac{1}{2}} / 2, \pm\left(3\left[1-\alpha^{2}\right]\right)^{\frac{1}{3}} / 2, \pm \alpha\right),\left(-\left[1-\alpha^{2}\right]^{\frac{1}{3}}, 0, \pm \alpha\right)$, where $\alpha$ is the remaining degree of freedom.

Likewise, when $n=11$ it becomes graphically clear that the arrangement is five points on the equator and three in each of the hemispheres, symmetric with respect to the equator $(z=0)$ and meridian $(y=0)$, yielding the configuration 
$(-1,0,0),\left(-\alpha_{1}, \pm\left(1-\alpha_{1}^{2}\right)^{\frac{1}{2}}, 0\right),\left(\alpha_{2}, \pm\left(1-\alpha_{2}^{2}\right)^{\frac{1}{2}}, 0\right),\left(-\alpha_{3}, \pm \alpha_{4}, \pm\left(1-\alpha_{3}{ }^{2}-\alpha_{4}^{2}\right)^{\frac{1}{2}}\right)$, $\left(\left(1-\alpha_{5}^{2}\right)^{\frac{1}{2}}, 0, \pm \alpha_{5}\right)$, with five degrees of freedom $\alpha_{i}$.

Thus we observe the final configuration for $n=9(n=11)$ and regenerate taking initially $\alpha=.699929\left(\alpha_{1}=.505694, \alpha_{2}=.507286, \alpha_{3}=.089001, \alpha_{4}=492006\right.$, $\left.\alpha_{5}=.571672\right)$. These are crude guesses based on averages of diagonal lengths. In fact $V$ now increases to 25.76028061 (40.64744085) which brings us back to the twenty-third (eleventh) step of the descent process! We are further ahead, however, by virtue of the new self-preserving symmetry. In fact in five (thirty) additional circuits of the main loop we find $V$ has come to the even lower value $25.75998651(40.59645048)$ probably correct to eight decimal places at which point the particle positions change by $\xi<10^{-5}=\epsilon$. The final values of the coordinates of the configuration are given by $\alpha=.703648\left(\alpha_{1}=.515358, \alpha_{2}=.552626\right.$, $\left.\alpha_{3}=.168322, \alpha_{4}=.488923, \alpha_{5}=.591930\right)$, probably correct to five decimal places, in a total of about 15(60) minutes of computing time.

The author is indebted to G. Pólya and J. L. Ullman for illuminating discussions and to Don Hart and George Ryckman of the General Motors Technical Center at Warren, Michigan for kindly making the IBM 701 available.

Department of Mathematics

HaRvey Cohn

Washington University

St. Louis, Missouri

1. L. FöPPL, "Stabile Anordnungen von Elektronen im Atom," J. für die Reine Angew. Math., v. 141, 1912, p. 251-301.

\section{An Iterative Method for Taylor Expansion of Rational Functions, and Applications}

1. The iteration and some applications. A simple iterative procedure can be set up for determining the coefficients of the Taylor expansion of a rational function at any point in the complex plane other than one of the singularities of the function. This procedure would have many applications, particularly in the evaluation of inverse Laplace transforms. We shall discuss the general method first, and then some applications, followed by a discussion of truncation and round-off errors.

Suppose $f(s)$ is a rational function, that is,

$$
f(s)=\frac{p(s)}{q(s)}
$$

where $p(s)$ and $q(s)$ are polynomials. We shall assume that we have $p(s)$ and $q(s)$ expressed in powers of $s$, rather than in their factored form.

Now consider any point " $a$ " in the complex plane, with the restriction that $q(a) \neq 0$. Then by Taylor's theorem, within the circle of convergence around " $a$ ", we have

$$
f(s)=\frac{p(s)}{q(s)}=\alpha_{0}+\alpha_{1}(s-a)+\alpha_{2}(s-a)^{2}+\cdots
$$

